\title{
LA FAUNA NATIVA DE MÉXICO EN RIESGO Y LA NOM-059: ¿ESTÁN TODOS LOS QUE SON Y SON TODOS LOS QUE ESTÁN?
}

\author{
MEXICAN NATIVE FAUNA AT RISK AND THE NOM-059 LISTING. \\ ARE ALL THE CALLED THERE, AND ARE THOSE THERE CALLED?
}

\author{
MARÍA C. GARCÍA-AGUILAR, ${ }^{1, *}$ JAIMe LUÉVANO-ESPARZA² Y HoRACIO DE LA CUEVA ${ }^{2}$ \\ ${ }^{1}$ Departamento de Oceanología Biológica, Centro de Investigación Científica y de Educación Superior \\ de Ensenada, Baja California. Carretera Ensenada-Tijuana 3918, Zona Playitas, CP 22860, \\ Ensenada, B. C., México. \\ ${ }^{2}$ Departamento de Biología de la Conservación, Centro de Investigación Científica y de Educación Superior de \\ Ensenada, Baja California. Carretera Ensenada-Tijuana 3918, Zona Playitas, CP 22860, Ensenada, B. C., México \\ <gaguilar@cicese.mx>,<jluevano@cicese.mx>, <cuevas@cicese.mx>. \\ * Autor de correspondencia: <gaguilar@cicese.mx>. \\ Recibido: 14/03/2016; aceptado: 27/0272017 \\ Editor responsable: Ricardo Rodríguez Estrella.
}

García-Aguilar, M. C., Luévano-Esparza, J. y de la Cueva, H. (2017). La fauna nativa de México en riesgo y la NOM-059: ¿Están todos los que son y son todos los que están? Acta Zoológica Mexicana (n.s.) 33(2), 188-198.

RESUMEN. La Norma Oficial Mexicana 059 (NOM-059) es el instrumento normativo que identifica las especies o poblaciones de México en riesgo mediante la aplicación del Método de Evaluación de Riesgo de Extinción de Especies Silvestres de México (MER). Desde su primera publicación en 1994 la Norma ha sido usada como referencia para evaluar el estado de conservación y como herramienta en las políticas ambientales nacionales; sin embargo, ha sido fuertemente cuestionada por la aparente falta de criterios científicos. El objetivo de este trabajo fue evaluar la pertinencia del uso de la NOM-059 como referencia del estado de conservación de las especies nativas de México utilizando a los mamíferos como caso de estudio. Más del $40 \%$ de los mamíferos nativos de México están incluidos, pero solo el 10\% del total de especies listadas se han evaluado con el MER; solo estas especies cuentan con soporte técnico que justifique su inclusión. En la mayoría de los casos los criterios empleados para determinar la inclusión se desconocen, pero en algunos casos son incluso contradictorios a los objetivos y metodología establecidos en la misma Norma. Los resultados sugieren que los mamíferos no endémicos están sobrerrepresentados en la NOM-059, mientras que el riesgo de extinción de los mamíferos endémicos está subestimado. La evidencia pone de manifiesto que en su forma actual la NOM-059 no es la herramienta adecuada para evaluar el estado de conservación. Finalmente, presentamos una serie de recomendaciones que consideramos contribuyen a mejorar la NOM-059 y así fortalecer los esfuerzos de conservación del país.

Palabras clave: Conservación, especies en riesgo, mamíferos, Método de Evaluación de Riesgo de Extinción de Especies Silvestres de México, riesgo de extinción.
García-Aguilar, M. C., Luévano-Esparza, J., \& de la Cueva, H. (2017). Mexican native fauna at risk and the NOM-059 listing. Are all the called there, and are those there called? Acta Zoológica Mexicana (n.s.), 33(2), 188-198.

ABSTRACT. The Norma Oficial Mexicana 059 (NOM-059) is the Mexican legal instrument that identifies species or populations at risk throug the use of the Método de Evaluación de Riesgo de Extinción de Especies Silvestres de México (MER). From its first promulgation in 1994, the NOM-059 has been used as a reference to assess the conservation status, and as a tool on national environmental policies; however, it has been questioned for the lack of scientific criteria. We assess the relevance of the NOM-059 as a tool to assess the conservation status of Mexican native species by utilizing the mammals as a case study. More than $40 \%$ of native mammals are included in the list, though only $10 \%$ of the listed species had been evaluated with the MER, and have technical support to justify their inclusion. In most cases, criteria used to determine the species inclusion are unknown, in the other cases the criteria are contradictory with the objectives and methods stablished in the NOM-059. Non-endemic mammals are overrepresented in the list, while the extinction risk of the endemic mammals is underestimated. The evidence shows that in its current form the NOM-059 it is not an adequate tool to assess the species conservation status. Finally, we list a series of recommendations that would improve NOM-059 and strengthen conservation efforts in Mexico

Key words: Conservation, endangered species, mammalians, Método de Evaluación de Riesgo de Extinción de Especies Silvestres de México, risk of extinction. 


\section{INTRODUCCIÓN}

México es reconocido como uno de los 17 países megadiversos, que en su conjunto reúnen entre el 65 y 70\% de la riqueza mundial de especies (Mittermeier et al., 2005). Como parte de su estrategia para la conservación, el gobierno federal mexicano, a través de la Secretaría de Medio Ambiente y Recursos Naturales (SEMARNAT), publica la Norma Oficial Mexicana 059 SEMARNAT (NOM-059 de aquí en adelante) cuyos objetivos son identificar a las especies o poblaciones de México en riesgo, elaborar los listados correspondientes y establecer los criterios de inclusión, exclusión o cambio de categoría de riesgo mediante la aplicación del Método de Evaluación de Riesgo de Extinción de Especies Silvestres de México (MER) (SEMARNAT, 2010). En 1994 se publicó la primera versión de la NOM-059, que consideraba cuatro categorías de riesgo: en peligro de extinción (P), amenazada (A), rara (R) y sujeta a protección especial (Pr) (INE, 1994). Unos años después se publicó la segunda versión con algunos cambios (SEMARNAT, 2002). Se estableció el uso del MER para determinar la inclusión o exclusión de especies, así como las categorías de riesgo. Se eliminó la categoría de rara y todas las especies que originalmente fueron incluidas en esta categoría se listaron de manera precautoria como sujetas a protección especial; además, se incluyó la categoría de probablemente extinta en vida silvestre (E). La versión más reciente de la NOM-059 se publicó en 2010 e incluye la revisión y actualización del listado previo (SEMARNAT, 2010).

Desde su primera versión, la NOM-059 ha sido usada como referencia para evaluar el estado de conservación de vertebrados terrestres nativos (e.g., anfibios y reptiles, Macip-Ríos \& Casas-Andreu, 2008; aves, Altamirano González et al., 2003; mamíferos, Ceballos et al., 2002), así como en el desarrollo y aplicación de las políticas de conservación, por ejemplo en la elaboración de la lista de especies prioritarias para la conservación (D.O.F. 05/03/2014). Sin embargo, la veracidad de la NOM-059 ha sido fuertemente cuestionada por la falta aparente de criterios objetivos y científicos en el sistema de clasificación y el uso de términos subjetivos en las categorías de riesgo (De Grammont \& Cuarón, 2006), dando origen a una controversia (ver Cuarón \& De Grammont, 2007 y Soberón \& Medellín, 2007).

Nuestro objetivo fue evaluar la pertinencia del uso de la NOM-059 como referencia del estado de conservación de las poblaciones y especies nativas de México a través de la presentación de un caso de estudio. Para tal fin elegimos a los mamíferos por dos razones: 1 ) su alta diversidad en el país (>500 especies nativas), y 2) por estar ampliamente representados en la NOM-059. Realizamos el conteo de especies listadas y evaluadas con el MER en la versión de 2010 de la NOM-059, y se hizo una comparación con la versión 2012.2 de la Lista Roja de la Unión Internacional para la Conservación de la Naturaleza (Lista Roja de aquí en adelante), que es reconocida a nivel mundial como la fuente de información más confiable sobre el estado de conservación de las especies (Rodrigues et al., 2006).

\section{MATERIALES Y MÉTODOS}

Se conformó un listado de los mamíferos de México tomando la nomenclatura de Wilson y Reeder (2005). El listado se elaboró con base en literatura especializada (Hall, 1981; Ceballos et al., 2005; Ceballos \& Oliva, 2005, Ramírez-Pulido et al., 2005; Escalante et al., 2007; Ceballos \& Arroyo-Cabrales, 2012) y las bases de datos de la Comisión Nacional para el Conocimiento y Uso de la Biodiversidad (www.conabio.org.mx/informacion/mamiferos), Global Biodiversity Information Facility (www. gbif.org), Index of Mammalian Species (www.science. smith.edu/msi/) y NatureServe (www.natureserve.org/natureserve-network). Se contabilizó el número de especies incluidas en la NOM-059-2010 en cada categoría de riesgo y el número de especies reportadas como evaluadas con el MER.

Se comparó el listado de la NOM-059 con la Lista Roja. Es importante notar que al hacer esta comparación se debe tomar en cuenta que los alcances de cada lista dependen de la escala (Breininger et al., 1998; Czech \& Krausman, 1997): la NOM-059 está diseñada para aplicarse a nivel nacional, mientras que la Lista Roja está elaborada a nivel global. Por ello, el estado conservación de una especie a nivel global no necesariamente coincide con el estado de conservación de la misma especie a nivel nacional, excepto en el caso de las especies endémicas donde el efecto de escala se elimina (Gärdenfors, 2001). Por ello, en la comparación de los listados se separó a las especies no endémicas de las endémicas. La Lista Roja considera ocho categorías de riesgo: extinta (EX), extinta en vida silvestre (EW), en peligro crítico (CR), en peligro de extinción (EN), vulnerable (VU), casi amenazada (NT), en preocupación menor (LC), y datos deficientes para la evaluación (DD) (IUCN, 2012). En el caso de la NOM-059, las especies en riesgo incluye a las clasificadas como en peligro de extinción (P) y amenazada (A), y 
en el caso de la Lista Roja a las especies incluidas en las categorías CR, EN y VU.

Se utilizó el coeficiente kappa ( $k$ ), que evalúa la proporción de concordancias entre observadores (i. e., listados), una vez excluidas las concordancias atribuibles al azar (Cohen, 1960). El coeficiente tiene valores $-1 \leq k \leq 1$, siendo que $k<0$ significa discrepancias sistemáticas entre observadores (i. e., no atribuibles al azar), $k=0$ representa concordancias atribuibles al azar, y $k>0$ concordancias entre observadores no atribuibles al azar. Se contrastó la hipótesis nula $\mathrm{H}_{0}: k=0$ a un nivel de significancia $\alpha=0.05$. La hipótesis nula puede rechazarse cuando 0 no está contenido dentro del intervalo de confianza $k \pm 1.96 * S E(k)$, donde $S E(k)$ es el error estándar de $k$ (Fleiss et al., 1979). La fuerza de la concordancia puede expresarse cualitativamente con la escala de Landis \& Koch (1977): valores de 0.0 a 0.20 representan una concordancia pobre, de 0.21 a 0.40 concordancia débil, de 0.41 a 0.60 concordancia moderada, de 0.61 a 0.80 concordancia alta, y de 0.81 a 1.00 concordancia casi perfecta.

\section{RESULTADOS Y DISCUSIÓN}

El número de especies que fueron consideradas en este estudio fue de 516, incluidas en 13 órdenes; 157 especies son endémicas (Cuadro 1). Doscientas catorce especies (41\% del total) están incluidas en la NOM-059, de las cuales ocho se clasifican como probablemente extintas en medio silvestre (Cuadro 1). Sin embargo, en la lista los términos extinción (i.e., proceso irreversible por el cual una especie deja de existir) y erradicación del Territorio Nacional (i.e., hay evidencia de la existencia de individuos vivos o de poblaciones fuera de México) se usan indistintamente. En consecuencia, se le asigna el mismo estatus de conservación a cinco especies extintas, los roedores endémicos Neotoma anthonyi, $N$. bunkeri, Oryzomys nelsoni y Peromyscus pembertoni (Cricetidae) y la foca monje del Caribe Monachus tropicalis (Phocidae: Carnivora), a una especie probablemente extinta, la rata canguro de San Quintín Dipodomys gravipes (Rodentia: Heteromyidae), y a dos especies erradicadas, el lobo $\mathrm{Ca}$ nis lupus (Canidae: Carnivora) y el oso pardo Ursus arctos (Ursidae: Carnivora).

Ciento doce especies (22\%) de los mamíferos de México están incluidos en las categorías en peligro de extinción (P) y amenazada (A) (Cuadro 1). Esto significa que de acuerdo con la NOM-059 una de cada cuatro especies vivas está en riesgo, pero como señalan De Grammont \& Cuarón (2006), las definiciones de estas dos categorías contienen elementos subjetivos (e.g., "disminuido drásticamente” y “corto o mediano plazo”), lo que

Cuadro 1. Diversidad de mamíferos de México y número de especies listadas en las categorías de riesgo de la NOM-059-2010. Entre paréntesis se muestra el número de especies evaluadas con el Método de Evaluación de Riesgo de Extinción de Especies Silvestres de México (MER).

Categorías de riesgo: $\mathrm{E}=$ probablemente extinta en medio silvestre, $\mathrm{P}=$ en peligro de extinción, $\mathrm{A}=$ amenazada, $\mathrm{Pr}=$ sujeta a protección especial, $\mathrm{NL}=$ no listadas.

\begin{tabular}{|c|c|c|c|c|c|c|c|c|c|c|c|c|c|}
\hline \multirow[b]{2}{*}{ Orden } & \multicolumn{6}{|c|}{ Especies no endémicas } & \multicolumn{6}{|c|}{ Especies endémicas } & \multirow[b]{2}{*}{ TOTAL } \\
\hline & $\mathbf{E}$ & $\mathbf{P}$ & $\mathbf{A}$ & Pr & NL & Suma & $\mathbf{E}$ & $\mathbf{P}$ & A & Pr & NL & Suma & \\
\hline Didelphimorphia & & $1(1)$ & $2(2)$ & & 4 & $7(3)$ & & & & & 1 & 1 & $8(3)$ \\
\hline Sirenia & & 1 & & & & 1 & & & & & & & 1 \\
\hline Cingulata & & 1 & & & 1 & 2 & & & & & & & 2 \\
\hline Pilosa & & 2 & & & & 2 & & & & & & & 2 \\
\hline Primates & & 3 & & & & 3 & & & & & & & 3 \\
\hline Rodentia & & 4 & 11 & 5 & 97 & 117 & $5(1)$ & $6(1)$ & $21(1)$ & 23 & 56 & $111(3)$ & $228(3)$ \\
\hline Lagomorpha & & & & & 8 & 8 & & $5(2)$ & & 1 & 1 & $7(2)$ & $15(2)$ \\
\hline Soricomorpha & & 2 & $4(2)$ & 1 & 7 & $14(2)$ & & & $8(5)$ & 7 & 6 & $21(5)$ & $35(7)$ \\
\hline Chiroptera & & 1 & $16(1)$ & 16 & 93 & $126(1)$ & & 3 & $1(1)$ & 1 & 9 & $14(1)$ & $140(2)$ \\
\hline Carnivora & 3 & 7 & 6 & $4(1)$ & 13 & $33(1)$ & & 1 & 1 & & & 2 & $35(1)$ \\
\hline Perissodactyla & & 1 & & & & 1 & & & & & & & 1 \\
\hline Artiodactyla & & $3(1)$ & & 1 & 5 & $9(1)$ & & & & & & & $9(1)$ \\
\hline Cetacea & & & & $35(2)$ & 1 & $36(2)$ & & $1(1)$ & & & & $1(1)$ & 37 (3) \\
\hline TOTAL & 3 & $26(2)$ & 39 (5) & $62(3)$ & 229 & $359(10)$ & $5(1)$ & $16(4)$ & $31(7)$ & 32 & 73 & $157(12)$ & $516(22)$ \\
\hline
\end{tabular}


permite la interpretación personal e imposibilita la discusión objetiva sobre la situación real de estas especies. La categoría sujeta a protección especial (Pr) es donde se incluye al mayor número de especies (44\% del total de especies listadas, Cuadro 1). Sin embargo, la definición de esta categoría es ambigua ya que cualquier especie puede "llegar a encontrarse amenazada por factores que inciden negativamente en su viabilidad" (SEMARNAT, 2010), por lo que todas las especies vivas que no estén en riesgo ( $\mathrm{P}$ o $\mathrm{A}$ ) tienen cabida. Además, como incluye explícitamente componentes de manejo ("se determina la necesidad de propiciar su recuperación y conservación”), aspecto señalado también por De Grammont \& Cuarón (2006), entra en el campo de acción de otro instrumento jurídico, la Ley General de Vida Silvestre.

Veintidós especies aparecen evaluadas con el MER (Cuadro 1); es decir, solamente el 10\% de los mamíferos listados cuenta con soporte técnico que justifique su inclusión y categorización, a pesar de que la Norma exige la documentación que demuestre el riesgo de extinción a través de la correcta aplicación del MER (inciso 6.1 de la versión 2010). En 2006 Low Pfeng reportó que hasta ese año menos del $10 \%$ del total de especies listadas habían sido evaluadas, nuestros resultados muestran que la tendencia no ha cambiado. Entre las características deseables del MER es que las variables que emplea son relativamente fáciles de obtener, pero al mismo tiempo son lo suficiente adecuadas para mostrar el estado de conservación; además, es flexible cuando la información es escasa o cuando los datos son de diferente calidad y tipo (Sánchez et al., 2007). Dadas estas ventajas del método, es difícil entender el por qué tan pocas especies se han evaluado, pero también pone en duda bajo qué criterios se han incluido más del 37\% de los mamíferos de México en la lista. Dado que la NOM-059 es una referencia para el gobierno mexicano en sus políticas ambientales, la evaluación del estado de conservación de las especies nativas de México debe ser considerada una acción prioritaria en términos de la conservación de la biodiversidad.

El número de especies no endémicas consideradas en riesgo de la NOM-059 es notablemente mayor al reportado en la Lista Roja, 65 vs. 17, respectivamente; además, las especies listadas no son las mismas (Cuadro 2; Anexo 1). De las 359 especies no endémicas, ambas listas consideran en riesgo a seis especies, mientras que el estatus de conservación de 70 es diferente (Cuadro 2). El coeficiente mostró que la concordancia entre listas es pobre $(k$ $=0.08)$ y, dado que la hipótesis nula no se rechazó, las concordancias observadas pueden ser atribuibles al azar
Cuadro 2. Comparación del número de especies no endémicas consideradas en riesgo en los listados de la NOM-059-2010 1 y la versión 2012 de Lista Roja de la IUCN², y coeficiente de kappa (k). $S E(k)=$ error estándar, IC 95\% = intervalo de confianza al 95\%.

\begin{tabular}{clccc}
\hline & & \multicolumn{3}{c}{ IUCN } \\
\cline { 3 - 5 } & & En riesgo & Otra categoría & Suma \\
\hline NOM-059 & En riesgo & 6 & 59 & 65 \\
& Otra categoría & 11 & 283 & 294 \\
& Suma & 17 & 342 & 359 \\
& $k$ & $S E(k)$ & IC 95\% & \\
& 0.08 & 0.10 & $-0.12-0.27$ & \\
\hline
\end{tabular}

${ }^{1}$ Incluye a las especies clasificadas en peligro de extinción (P) y amenazada (A).

${ }^{2}$ Incluye a las especies clasificadas en peligro crítico (CR), en peligro de extinción (EN) y vulnerable (VU).

(Cuadro 2). Estos resultados podrían deberse a la escala de cada lista. De las discrepancias observadas, hay 59 especies que la NOM-059 considera que están en riesgo, pero la Lista Roja considera que a nivel global (i.e., en todo su rango de distribución) no lo están (Cuadro 2). Esto significa que el estado de conservación de las poblaciones de estas especies en México está deteriorado en comparación al estado de conservación global. Por otra parte, hay 11 especies que a nivel global la Lista Roja clasifica en riesgo pero no la NOM-059 (Cuadro 2), lo que implica que aparentemente las poblaciones en México de estas especies se encuentran en mejor estado que a nivel global. Sin embargo, estas interpretaciones pueden ser erróneas debido a que solamente el $2.5 \%$ de las especies no endémicas han sido evaluadas con el MER (ver Cuadro 1); es decir, la inclusión (o exclusión) y categorización carecen de soporte técnico que la justifique.

En el caso de las especies endémicas era de esperarse una buena concordancia entre las listas debido a que el efecto de escala se elimina; sin embargo, esto no sucedió. Sorprendentemente, la Lista Roja enlista un mayor número de especies que la NOM-059, 80 vs. 47, respectivamente. El estatus de 39 especies coincide, pero el de 49 es diferente, por lo que el coeficiente mostró una concordancia débil $(k=0.38)$ no atribuible al azar (Cuadro 3). La NOM-059 considera que 40 especies endémicas (principalmente roedores) no están en riesgo, mientras que la Lista Roja las enlista en riesgo (Cuadro 3), con una alta proporción (ca. 75\%) en peligro crítico y en peligro de extinción (Anexo 1). Al contrario, la Lista Roja no considera en riesgo a ocho especies que la NOM-059 sí enlista (Cuadro 3, Anexo 1). Un aspecto importante que debe recalcarse es el hecho de que, a pesar de que la Lista Roja 
Cuadro 3. Comparación del número de especies endémicas consideradas en riesgo en los listados de la NOM-059-2010 y la versión 2012 de Lista Roja de la IUCN ${ }^{2}$, y coeficiente de kappa ( $k$ ). $S E(k)=$ error estándar de kappa, IC 95\% = intervalo de confianza al 95\%.

\begin{tabular}{clccc}
\hline & \multicolumn{3}{c}{ IUCN } \\
\cline { 3 - 5 } & & En riesgo & Otra categoría & Suma \\
\hline NOM-059 & En riesgo & 39 & 8 & 47 \\
& Otra categoría & 41 & 69 & 110 \\
& Suma & 80 & 77 & 157 \\
& $k$ & $S E(k)$ & IC 95\% & \\
& 0.38 & 0.07 & $0.24-0.52$ & \\
\hline
\end{tabular}

${ }^{1}$ Incluye a las especies clasificadas en peligro de extinción (P) y amenazada (A).

${ }^{2}$ Incluye a las especies clasificadas en peligro crítico (CR), en peligro de extinción (EN) y vulnerable (VU).

tiene criterios de evaluación y clasificación más estrictos que la NOM-059, el 95\% de las especies endémicas de México han sido evaluadas por los expertos de IUCN, pero solamente $7.6 \%$ han sido evaluadas con el MER (ver Cuadro 1). El conjunto de las diferencias sugiere que la NOM-059 subestima el número de especies endémicas en riesgo, dejándolas de lado en los planes de recuperación y conservación de la biodiversidad.

\section{Consideraciones finales y recomendaciones}

Las listas de especies en riesgo se construyen y publican para ofrecer un entendimiento cualitativo y sencillo del riesgo de extinción que ayude a definir las prioridades en las políticas de manejo y conservación (Possingham et al., 2002), por lo que su elaboración es un paso importante para ayudar a combatir la pérdida de biodiversidad. En México, la NOM-059 es el instrumento jurídico que aborda la evaluación del riesgo de extinción (Sánchez et al., 2007), y por lo tanto, su conformación debería estar sustentada exclusivamente en criterios científicos (i.e., el MER), la realidad es otra.

A pesar del alto número de especies listadas, la proporción de especies evaluadas es sumamente baja y en muchos casos los criterios empleados para determinar su inclusión y categorización se desconocen; en otros casos son incluso contradictorios a los objetivos y metodología establecidos en la misma Norma. Un claro ejemplo de esto es el caso de los cetáceos, que fueron incorporados no porque se hubiera demostrado el riesgo de extinción de todas estas especies que habitan en aguas mexicanas aplicando el MER, sino porque el gobierno mexicano las considera de "especial interés para fortalecer las medidas de ordenamiento pesquero y por los tratados internacionales que México ha firmado para su protección” (SEMARNAT, 2002). Estas situaciones sugieren que el listado es el resultado de una elección a priori de las especies que deben ser incluidas, tal como ha sucedido con otras listas (Hilton-Taylor et al., 2000). En el caso de la NOM-059, esta elección corresponde a la SEMARNAT, tal como se establece en el inciso 8.7, que textualmente dice que "la determinación de la inclusión, exclusión o cambio de categoría de riesgo de las especies propuestas, quedará a cargo de la SEMARNAT".

La comparación con la Lista Roja sugiere que los mamíferos no endémicos podrían estar sobrerrepresentados, y tal vez su inclusión obedezca a intereses personales de investigadores, manejadores o políticos (como el caso de los cetáceos), o bien ser un reflejo de la tendencia a incluir especies grandes y carismáticas (Metrick \& Weitzman ,1996). El caso de las especies endémicas es aún más preocupante porque la NOM-059 (y por lo tanto, el gobierno mexicano) subestima el riesgo de extinción, lo que representa un problema en el establecimiento de las prioridades de conservación.

Debido a la escasa base científica en la conformación de la NOM-059 y a las inclusiones ajenas al riesgo de extinción, es imposible determinar si todas las poblaciones o especies en riesgo se encuentran listadas, ¿Están todos los que son? O si todas las poblaciones o especies listadas están realmente en riesgo, ¿Son todos los que están? Por lo que consideramos que en su forma actual la NOM-059 no es una herramienta adecuada para evaluar el estado de conservación.

Más que una crítica, este trabajo es una revisión independiente que aporta información e ideas para mejorar la estructura y el contenido de la NOM-059. El tema que debe ocuparnos tanto a investigadores como a agencias gubernamentales y público en general es la revisión y mejora de los mecanismos empleados para determinar la inclusión, exclusión y categorización de las especies. Por ello, presentamos las siguientes recomendaciones:

- Excluir de la lista a las especies extintas y diferenciar entre especies probablemente extintas de especies erradicadas.

- Eliminar términos subjetivos de las categorías En Peligro de Extinción (P) y Amenazada (A) sustituyéndolos con términos objetivos basados en las tendencias poblacionales y distribución de la especie.

- Eliminar la categoría de Sujeta a Protección Espe- 
cial (Pr) y considerar a todas las especies vivas que no estén en riesgo (categorías $\mathrm{P}$ y A) como parte importante de la dinámica de los sistemas que habitan.

- Incorporar la categoría Información Insuficiente (II) para todas aquellas especies de las cuáles se carezca de la información poblacional básica, por lo que no sea posible evaluarlas con el MER.

- Incentivar a los investigadores e instituciones para evaluar de manera independiente y objetiva a las especies y poblaciones a través de la aplicación del MER.

- Hacer el proceso de inclusión y categorización de las especies transparente mostrando al público los resultados del MER, así como el nombre del evaluador y la fecha.

- Eliminar el inciso 8.7 de la NOM-059 con la finalidad de que la lista sea el resultado de un ejercicio científico e independiente que muestre el estado de conservación de las especies y poblaciones.

- Ampliar el proceso de determinación de la categoría de las especies para que la SEMARNAT ratifique, no califique, la categoría de la especie.

- En el caso particular de las especies endémicas, adoptar de manera precautoria la categorización de la Lista Roja de la Unión Internacional para la Conservación de la Naturaleza hasta que se realicen las evaluaciones correspondientes mediante el MER.

- Propiciar la investigación y conservación de las especies que no estén en riesgo para evitar que suban de categoría.

AGRADECIMIENTOS. Agradecemos a tres revisores anónimos por sus comentarios y sugerencias.

\section{LITERATURA CITADA}

Altamirano González, M. A., Guzmán Hernández, J., Martin Gómez, M. F. \& Domínguez Velázquez, L. E. (2003). Un método para la selección de aves bioindicadoras con base en sus posibilidades de monitoreo. Huitzil, 4, 10-16.

Breininger, D. R., Barkaski, M. J., Smith, R. B., Oddy, D. M. \& Provancha, J. A. (1998). Prioritizing wildlife taxa for biological diversity conservation at the local scale. Environmental Management, 22, 315-321.

Ceballos, G. \& Arroyo-Cabrales, J. (2012). Lista actualizada de los mamíferos de México 2012. Revista Mexicana de Mastozoología (n. e.), 2, 27-80.

Ceballos, G., Arroyo-Cabrales J. \& Medellín, R. A. (2002). Mamí- feros de México. Pp. 377-413. In: G. Ceballos \& J. A. Simonetti (Eds.). Diversidad y conservación de los mamíferos neotropicales. CONABIO-UNAM, México D. F.

Ceballos, G., Arroyo-Cabrales, J., Medellín, R. A. \& DomínguezCastellanos, Y. (2005). Lista actualizada de los mamíferos de México. Revista Mexicana de Mastozoología, 9, 21-71.

Ceballos, G. \& Oliva, G. (2005). Los mamíferos silvestres de México. CONABIO, México D. F., 986 pp.

Cohen, J. (1960). A coefficient of agreement for nominal scales. Educational and Psychological Measurement 20, 37-46.

Cuarón, A. D. \& De Grammont, P. C. (2007). Shortcomings of threatened species categorization systems: replay to Soberón and Medellín. Conservation Biology, 21, 1368-1370.

Czech, B. \& Krausman, P. R. (1997). Distribution and causation of species endangerment in the United States. Science, 277, 11161117.

De Grammont, P. C. \& Cuarón, A. D. (2006). An evaluation of threatened species categorization systems used on the American continent. Conservation Biology, 20, 14-27.

D.O.F. 05/03/2014. Acuerdo por el que se da a conocer la lista de especies y poblaciones prioritarias para la conservación. Estados Unidos Mexicanos.- Secretaría de Medio Ambiente y Recursos Naturales. México, D. F., México.

Escalante, T., Sánchez-Cordero, V., Morrone, J. J. \& Linaje, M. (2007). Deforestation affects biogeographical regionalization: a case study contrasting potential and extant distributions of Mexican terrestrial mammals. Journal of Natural History, 41, 964-984.

Fleiss, J. L., Cohen, J. \& Everitt, B. S. (1969). Large sample standard errors of kappa and weighted kappa. Psychology Bulletin 72, 323-237.

Gärdenfors, U. (2001). Classifying threatened species at national versus global levels. Trends in Ecology and Evolution, 16, 511-516.

Hall, E. R. (1981). The mammals of North America. 2 vols. John Wiley \& Sons, New York, 1300 pp.

Hilton-Taylor, C., Mace, G. M., Capper, D. R., Collar, N. J., Stuart, S. N., Bibby, C. J., Pollock, C. \& Thomsen, J. B. (2000). Assessment mismatches must be sorted out: they leave species at risk. Nature, 404, 541.

INE. (1994). Norma Oficial Mexicana NOM-059-ECIL-1994, que determina las especies y subespecies de flora y fauna silvestres terrestres y acuáticas en peligro de extinción, amenazadas, raras y las sujetas a protección especial, y que establece especificaciones para su protección. Diario Oficial de la Federación 16 mayo, 1994.

IUCN. (2012). IUCN Red List categories and criteria. Version 3.1. IUCN, Gland, $32 \mathrm{pp}$.

Landis, J. R. \& Koch, G. G. (1977). The measurement of observer agreement for categorical data. Biometrics 33, 159-174.

Low Pfeng, A. (2006). Especies en riesgo. Norma Oficial Mexicana NOM-059-SEMARNAT-2001: actualización 2006. Sociedad Ictiológica Mexicana, Publicaciones Ocasionales, 6, 16-28.

Macip-Ríos, R. \& Casas-Andreu, G. (2008). Los cafetales en México y su importancia para la conservación de anfibios y reptiles. Acta Zoológica Mexicana (n.s.), 24, 143-159.

Metrick, A. \& Weitzman, M. M. (1996). Patterns of behavior in endangered species preservation. Land Economics 72, 1-16.

Mittermeier, R. A., Goettsch-Mittermeier, C. \& Robles-Gil, P. 
(2005). Megadiversity: Earth's biologically wealthiest nations. Cemex, México D. F., 501 pp.

Possingham, H. P., Andelman, S. J., Burgman, M. A., Medellín, R. A., Master, L. L. \& Keith, D. A. (2002). Limits to the use of threatened species lists. Trends in Ecology and Evolution, 17, 503-507.

Ramírez-Pulido, J., Arroyo-Cabrales, J. \& Castro-Campillo, A. (2005). Estado actual y relación nomenclatural de los mamíferos terrestres de México. Acta Zoológica Mexicana (n.s.), 21, 21-82.

Rodrigues, A. S. L., Pilgrim, J. D., Lamoreux, J. F., Hoffman, M., \& Brooks, T. M. (2006). The value of the IUCN Red List for conservation. Trends in Ecology and Evolution, 21, 71-76.

Sánchez, O., Medellín, R., Aldama, A., Goettsch, B., Soberón, J. \& Tambutti, M. (2007). Método de evaluación del riesgo de extinción de las especies silvestres de México (MER). SEMARNATUNAM-CONABIO, México D. F., 173 pp.
SEMARNAT. (2002). Norma Oficial Mexicana NOM-059-ECOL2001, Protección ambiental- Especies nativas de México de flora y fauna silvestres- Categorías de riesgo y especificaciones para su inclusión, exclusión o cambio- Lista de especies en riesgo. Diario Oficial de la Federación 06 marzo, 2002.

SEMARNAT. (2010). Norma Oficial Mexicana NOM-059-SEMARNAT-2010, Protección ambiental- Especies nativas de México de flora y fauna silvestres- Categorías de riesgo y especificaciones para su inclusión, exclusión o cambio- Lista de especies en riesgo. Diario Oficial de la Federación 30 diciembre, 2010.

Soberón, J. \& Medellín, R. A. (2007). Threatened species categorization systems. Conservation Biology, 21, 1368-1370.

Wilson, D. E., \& Reeder, D. M. (2005). Mammal species of the world. A taxonomic and geographic reference. The Johns Hopkins University Press, Baltimore. 2142 pp.

\section{ANEXO 1}

Concordancias y discrepancias entre las especies listadas en riesgo en la NOM-059-2010 y la versión 2012.2 de la Lista Roja de IUCN Categorías de riesgo NOM-059-2010: $\mathrm{E}$ = probablemente extinta en el medio silvestre, $\mathrm{P}=$ en peligro de extinción, $\mathrm{A}=$ amenazada, $\mathrm{Pr}=$ sujeta a protección especial.

Categorías de riesgo Lista Roja de la IUCN: EX = extinta, EW = extinta en vida silvestre, CR = en peligro crítico, EN = en peligro de extinción, $\mathrm{VU}=$ vulnerable, $\mathrm{NT}$ = casi amenazada, LC = en preocupación menor, DD = datos deficientes para la evaluación.

MER = Método de Evaluación de Riesgo de Extinción de Especies Silvestres de México.

\subsection{Concordancias entre las listas}

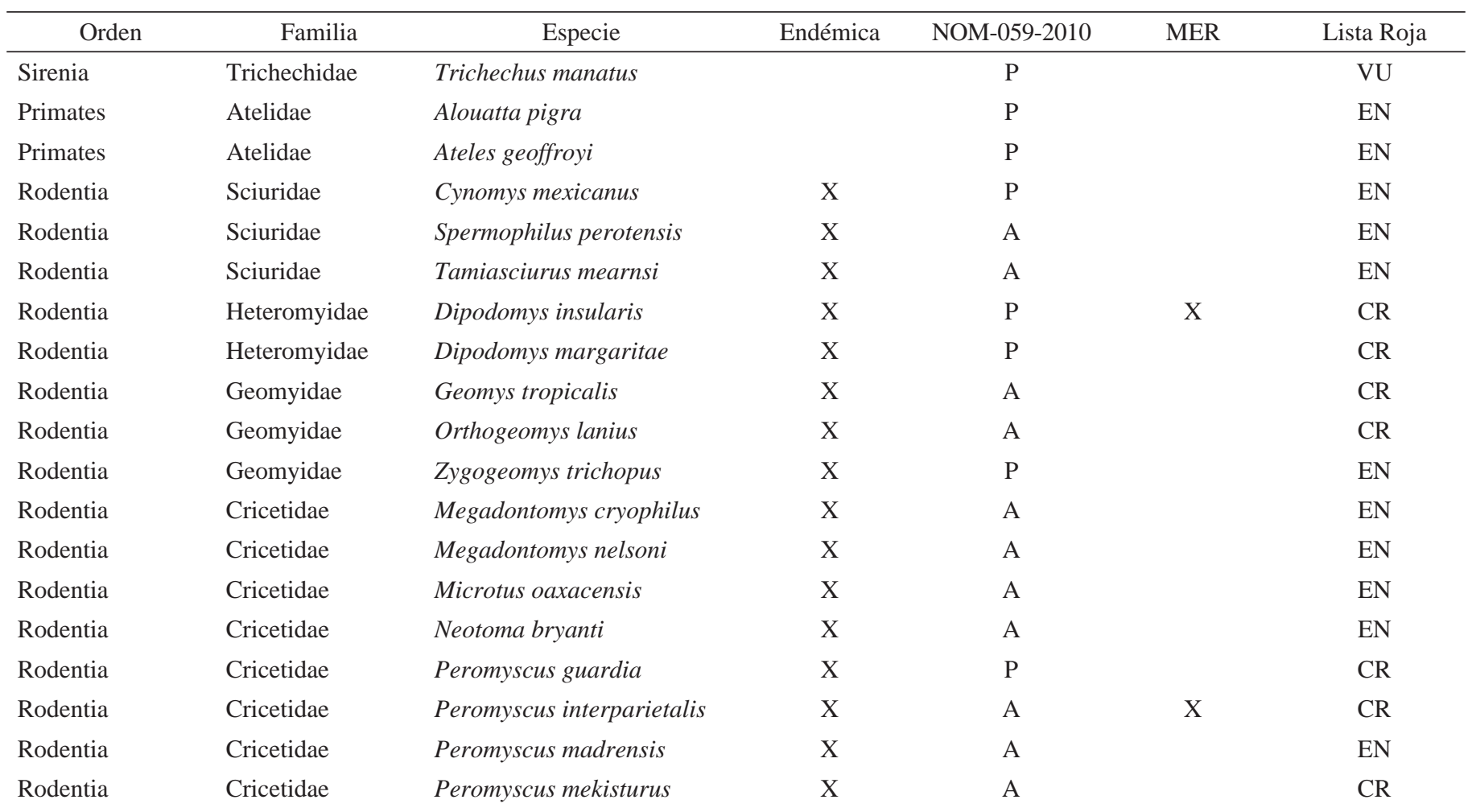




\begin{tabular}{|c|c|c|c|c|c|c|}
\hline Orden & Familia & Especie & Endémica & NOM-059-2010 & MER & Lista Roja \\
\hline Rodentia & Cricetidae & Peromyscus pseudocrinitus & $\mathrm{x}$ & A & & $\mathrm{CR}$ \\
\hline Rodentia & Cricetidae & Peromyscus sejugis & $\mathrm{X}$ & A & & EN \\
\hline Rodentia & Cricetidae & Peromyscus slevini & $\mathrm{X}$ & A & & CR \\
\hline Rodentia & Cricetidae & Peromyscus stephani & $\mathrm{x}$ & A & & CR \\
\hline Rodentia & Cricetidae & Reithrodontomys spectabilis & $\mathrm{x}$ & A & & CR \\
\hline Rodentia & Cricetidae & Tylomys bullaris & $\mathrm{x}$ & A & & CR \\
\hline Rodentia & Cricetidae & Xenomys nelsoni & $\mathrm{x}$ & A & & EN \\
\hline Lagomorpha & Leporidae & Lepus flavigularis & $\mathrm{x}$ & $\mathrm{P}$ & & EN \\
\hline Lagomorpha & Leporidae & Romerolagus diazi & $\mathrm{x}$ & $\mathrm{P}$ & & EN \\
\hline Lagomorpha & Leporidae & Sylvilagus graysoni & $\mathrm{x}$ & $\mathrm{P}$ & $\mathrm{X}$ & EN \\
\hline Lagomorpha & Leporidae & Sylvilagus insonus & $\mathrm{x}$ & $\mathrm{P}$ & & EN \\
\hline Lagomorpha & Leporidae & Sylvilagus mansuetus & $\mathrm{x}$ & $\mathrm{P}$ & $\mathrm{X}$ & CR \\
\hline Soricomorpha & Soricidae & Notiosorex villai & $\mathrm{x}$ & A & $\mathrm{X}$ & VU \\
\hline Soricomorpha & Soricidae & Sorex macrodon & $\mathrm{x}$ & A & $\mathrm{X}$ & VU \\
\hline Soricomorpha & Soricidae & Sorex sclareti & $\mathrm{x}$ & A & $\mathrm{X}$ & CR \\
\hline Soricomorpha & Soricidae & Sorex stizodon & $\mathrm{x}$ & A & $\mathrm{x}$ & CR \\
\hline Chiroptera & Phyllostomidae & Leptonycteris curasoae & & A & & VU \\
\hline Chiroptera & Phyllostomidae & Leptonycteris nivalis & & A & & EN \\
\hline Chiroptera & Phyllostomidae & Musonycteris harrisoni & $\mathrm{x}$ & $\mathrm{P}$ & & VU \\
\hline Chiroptera & Vespertilionidae & Myotis planiceps & $\mathrm{x}$ & $\mathrm{P}$ & & EN \\
\hline Chiroptera & Vespertilionidae & Myotis vivesi & $\mathrm{x}$ & $\mathrm{P}$ & & VU \\
\hline Chiroptera & Vespertilionidae & Rhogeessa genowaysi & $\mathrm{x}$ & A & $\mathrm{x}$ & EN \\
\hline Carnivora & Mephitidae & Spilogale pygmaea & $\mathrm{x}$ & A & & VU \\
\hline Carnivora & Procyonidae & Procyon pygmaeus & $\mathrm{x}$ & $\mathrm{P}$ & & CR \\
\hline Perissodactyla & Tapiridae & Tapirus bairdii & & $\mathrm{P}$ & & EN \\
\hline Cetacea & Phocoenidae & Phocoena sinus & $\mathrm{x}$ & $\mathrm{P}$ & $\mathrm{X}$ & CR \\
\hline
\end{tabular}

\subsection{Discrepancias en las listas}

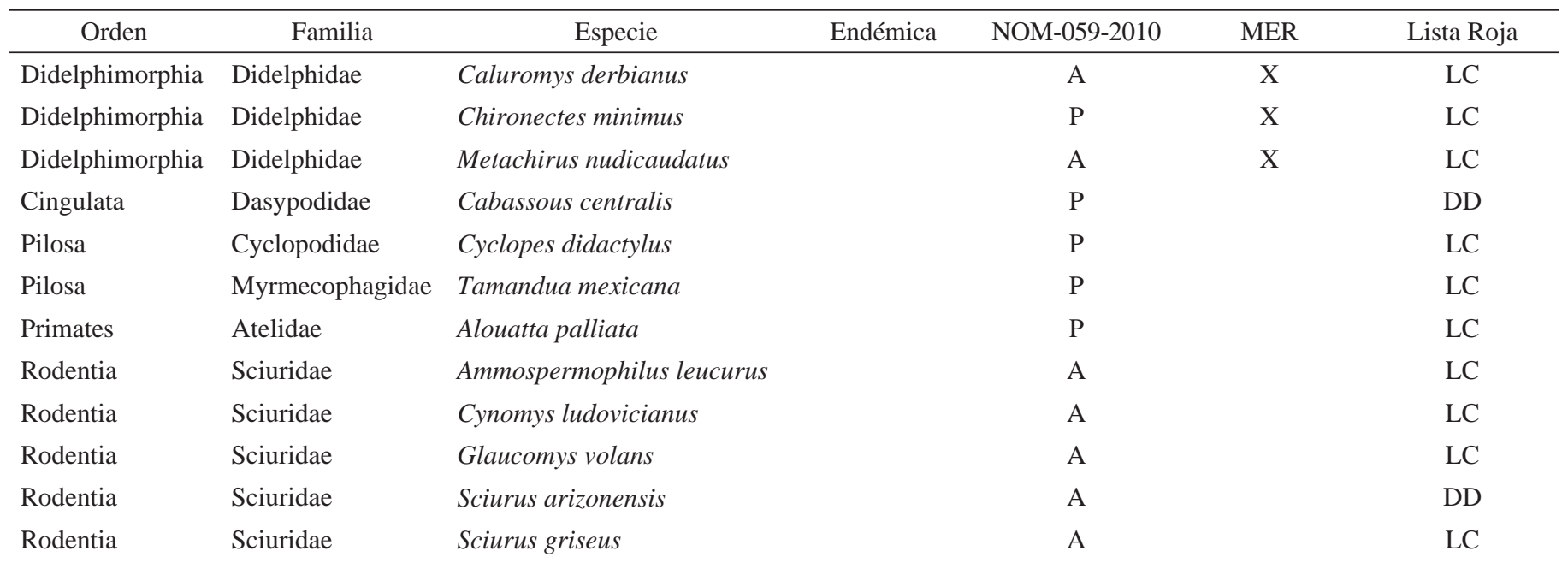




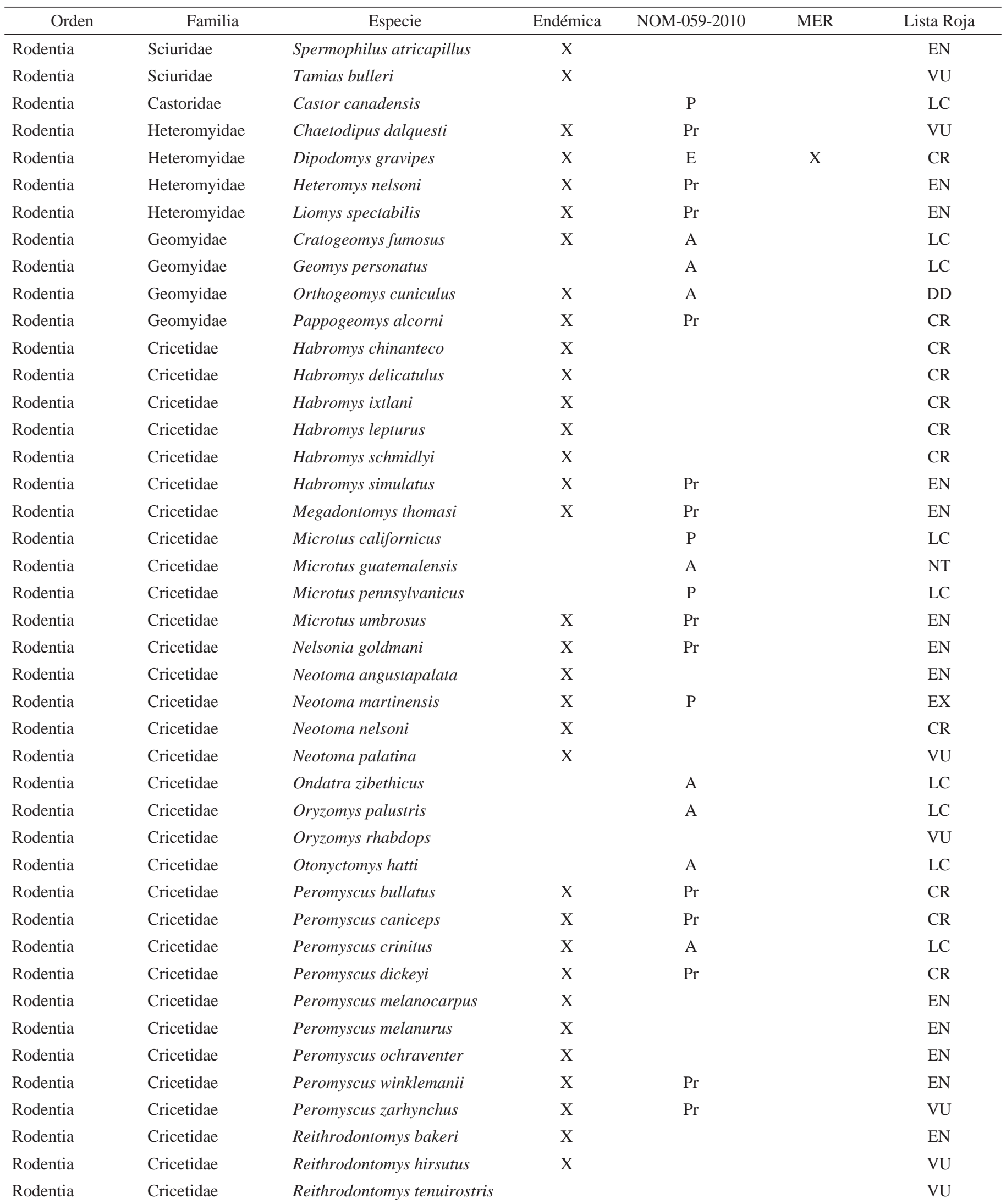




\begin{tabular}{|c|c|c|c|c|c|c|}
\hline Orden & Familia & Especie & Endémica & NOM-059-2010 & MER & Lista Roja \\
\hline Rodentia & Cricetidae & Rheomys mexicanus & $\mathrm{X}$ & $\operatorname{Pr}$ & & EN \\
\hline Rodentia & Erethizontidae & Sphiggurus mexicanus & & A & & $\mathrm{LC}$ \\
\hline Rodentia & Cricetidae & Sigmodon planifrons & $\mathrm{X}$ & & & EN \\
\hline Rodentia & Cricetidae & Tylomys tumbalensis & $\mathrm{X}$ & $\operatorname{Pr}$ & & CR \\
\hline Rodentia & Dasyproctidae & Dasyprocta mexicana & $\mathrm{X}$ & & & CR \\
\hline Lagomorpha & Leporidae & Sylvilagus robustus & & & & EN \\
\hline Soricomorpha & Soricidae & Cryptotis griseoventris & & & & VU \\
\hline Soricomorpha & Soricidae & Cryptotis magna & $\mathrm{X}$ & $\operatorname{Pr}$ & & VU \\
\hline Soricomorpha & Soricidae & Cryptotis nelsoni & $\mathrm{X}$ & $\operatorname{Pr}$ & & CR \\
\hline Soricomorpha & Soricidae & Notiosorex crawfordi & & A & & LC \\
\hline Soricomorpha & Soricidae & Notiosorex evotis & $\mathrm{X}$ & A & & $\mathrm{LC}$ \\
\hline Soricomorpha & Soricidae & Sorex arizonae & & $\mathrm{P}$ & & LC \\
\hline Soricomorpha & Soricidae & Sorex milleri & $\mathrm{X}$ & $\operatorname{Pr}$ & & VU \\
\hline Soricomorpha & Soricidae & Sorex monticolus & & A & $\mathrm{X}$ & $\mathrm{LC}$ \\
\hline Soricomorpha & Soricidae & Sorex orizabae & $\mathrm{X}$ & A & & LC \\
\hline Soricomorpha & Soricidae & Sorex veraecrusis & $\mathrm{X}$ & A & $\mathrm{X}$ & LC \\
\hline Soricomorpha & Soricidae & Sorex veraepacis & & A & $\mathrm{X}$ & $\mathrm{LC}$ \\
\hline Chiroptera & Phyllostomidae & Leptonycteris yerbabuenae & & & & VU \\
\hline Chiroptera & Phyllostomidae & Lonchorhina aurita & & A & & $\mathrm{LC}$ \\
\hline Chiroptera & Phyllostomidae & Lophostoma brasiliense & & A & & LC \\
\hline Chiroptera & Phyllostomidae & Lophostoma evotis & & A & & LC \\
\hline Chiroptera & Phyllostomidae & Macrophyllum macrophyllum & & A & & $\mathrm{LC}$ \\
\hline Chiroptera & Phyllostomidae & Micronycteris schmidtorum & & A & & $\mathrm{LC}$ \\
\hline Chiroptera & Phyllostomidae & Mimon cozumelae & & A & & LC \\
\hline Chiroptera & Phyllostomidae & Mimon crenulatum & & A & & $\mathrm{LC}$ \\
\hline Chiroptera & Phyllostomidae & Phylloderma stenops & & A & & LC \\
\hline Chiroptera & Phyllostomidae & Tonatia saurophila & & A & & LC \\
\hline Chiroptera & Phyllostomidae & Trachops cirrhosus & & A & & $\mathrm{LC}$ \\
\hline Chiroptera & Phyllostomidae & Vampyrum spectrum & & $\mathrm{P}$ & & NT \\
\hline Chiroptera & Mormoopidae & Pteronotus gymnonotus & & A & $\mathrm{X}$ & $\mathrm{LC}$ \\
\hline Chiroptera & Vespertilionidae & Myotis findleyi & $\mathrm{X}$ & & & EN \\
\hline Chiroptera & Vespertilionidae & Myotis peninsularis & $\mathrm{X}$ & & & EN \\
\hline
\end{tabular}




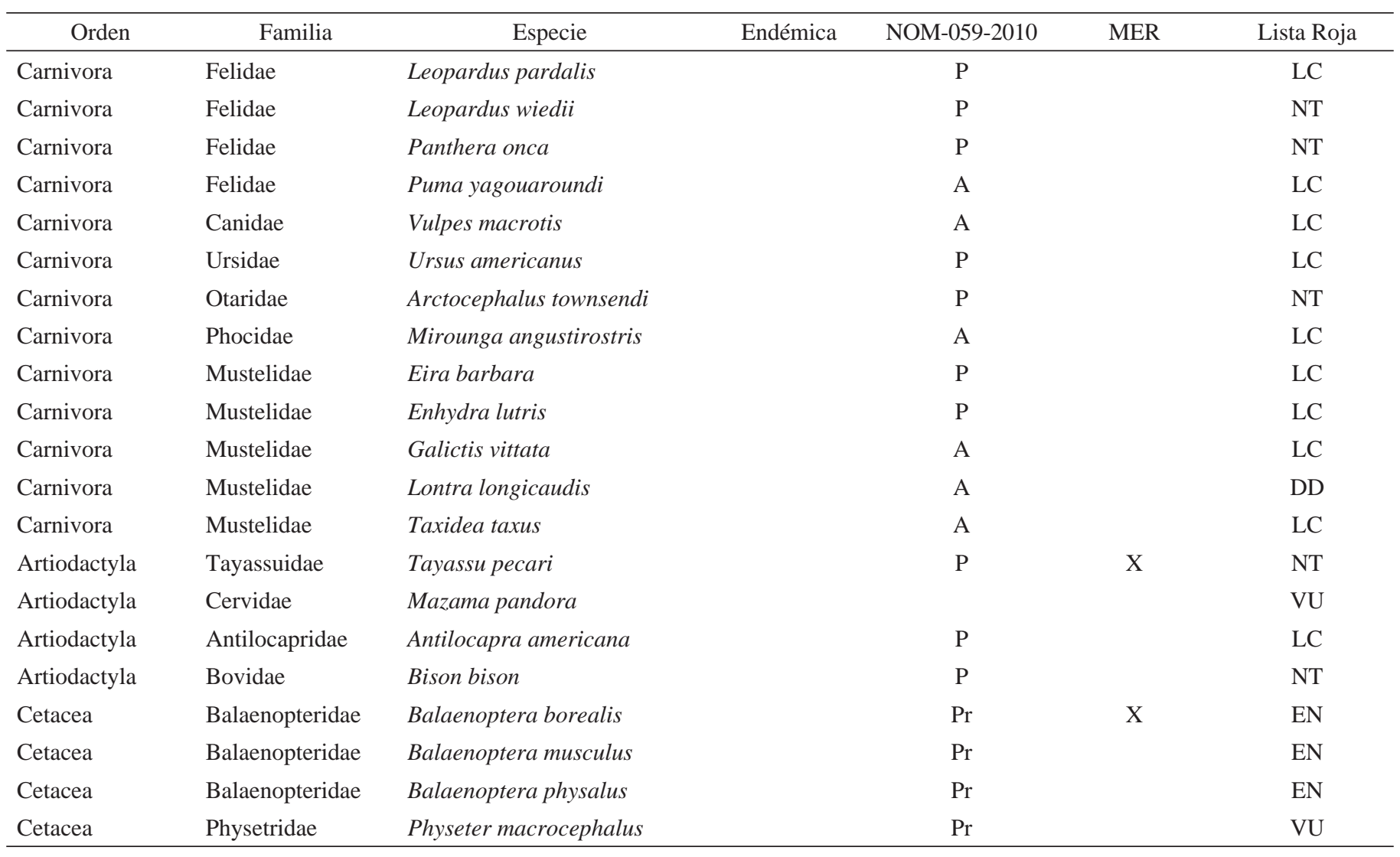

